\title{
STATE OF THE ART: INTEGRATING SERVICES FOR MEGA EVENTS
}

\author{
Jorge R. B. Garay \\ Gustavo M. Calixto \\ Alexandre M. De Oliveira \\ Marcelo K. Zuffo \\ University of São Paulo, POLI/USP, São Paulo, SP, Brazil \\ Interdisciplinary Center in Interactive Technologies, CITI/USP, SP, Brazil
}

\begin{abstract}
This paper describes the state of the art of a simplified model for integration of services in mega events. The project context, which was financed by the National Research Council - CNPq, is divided into six major areas or functional groups: Urban Mobility, Tourism, Airports, Security, Energy and Telecommunications. The proposal is described in layers, as an infrastructure model of integration and services evaluation, describing its main layers and interaction processes. The proposal described in this paper is restricted to the telecommunication subproject; however, in the modeling of the scenario for the study case, it was necessary to consider requirements and variables that are common to all subprojects. In the specification process of these requirements, we noticed important processes that interact with some sectors in the city of São Paulo; however, in the mean time, we noticed some failures regarding the integration and collaboration performed by administrative elements of stadiums as a main focus in the
\end{abstract}

Manuscript first received/Recebido em: 25/02/2014 Manuscript accepted/Aprovado em: 27/06/2014

Address for correspondence / Endereço para correspondência

Jorge Rodolfo Beingolea Garay, is B.Sc. in Computing (2004) Universidad Inca Garcilaso de La Vega. M.Sc. in Electrical Engineering (2007) - University of São Paulo, and PhD. in Electrical Engineering (2012) - University of São Paulo. He is currently a PAD Group Researcher in the Integrated Systems Laboratory (LSI - EPUSP) and of Interdisciplinary Center in Interactive Technologies (CITI) at USP. He has experience in Electrical Engineering, with emphasis on Wireless Sensor Networks, Wireless Communication, Wireless Networks and Pervasive Computing, Computer Architectures, Complex Systems, Internet of Things, Service-Based Architectures, and Cyber-Physical System. E-mail: jorge@1si.usp.br

Gustavo M. Calixto, is B.Sc. in Computer Technology (2004) and M.Sc. for University of Campinas. and PhD student in the University of São Paulo. He is currently a Researcher in Interdisciplinary Center in Interactive Technologies (CITI) at USP, with experience in Electrical Engineering, with emphasis on Digital TV. E-mail: calixto@lsi.usp.br

Alexandre M. De Oliveira, is B.Sc. in Electrical Engineering with Computer emphasis - Catholic University of Santos (2008), M.Sc. in Electrical Engineering (2012) - University of São Paulo, and PhD student in the University of São Paulo. He is currently Group PAD Researcher in the Integrated Systems Laboratory (LSI) at EPUSP, with experience in Electrical Engineering, with emphasis on VLSI Design, UWB I-Radar, Timed-array propagation, Microwave and Electromagnetism, and Numerical Methods and simulations for Electromagnetism. E-mail: amanicoba@pad.lsi.usp.br

Marcelo Knörich Zuffo, Graduated in Electrical Engineer (1989) M.Sc. in Electrical Engineering (1993) and PhD. in Electrical Engineering (1997) at University of São Paulo. He is full professor at University of Sao Paulo since 2006 at the electronics systems department. He is the chief of R\&D activities of the Laboratory for Integrated Systems and of Interdisciplinary Center in Interactive Technologies (CITI) at USP focused on interactive technologies, digital health, high performance computing, virtual reality, graphics computing and visualization. He is member of ACM and of Brazilian Digital Television System Forum since its foundation in 2007. E-mail: mkzuffo@ 1si.usp.br 
mega event and part of our case study. Currently, the proposal is in its first version as open software for the evaluation of the services quality and the mega event infrastructure through the use of performance indicators.

Keywords: Mega Events; Infrastructure Services; Framework; KPI; Cloud.

\section{INTRODUCTION}

Brazil, in the past few years, has been preparing itself to support worldwide events (Mega Events), such as the FIFA World Cup in 2014 and the Olympics in 2016. Both events have the challenge of providing the necessary infrastructure for the proper conduct of the activities that will be performed. Regarding the FIFA World Cup, in which there is a distribution of the host cities in all regions of the country, the logistics of transportation and the offering of basic information to the participating community (athletes, spectators, employees) are essential. The monitoring of services (the entire infrastructure to ensure the good progress of the event) offered to the participating community can result in the generation of indicators, which, in the future, can contribute to the evaluation of lessons learned and also to the analysis of the event's legacy.

The use of mobile devices for easy access and information sharing can be considered as a concept in the exponential growth phase. Currently, the number of users of these mobile devices is increasing (N.Eagle, 2005), as well as the extent of features that, today, these embedded devices offer. The innovation rate of the functions grows along with the market of applications that allow user location tracking (Rose, 2011), multimedia resource sharing, multi-user games (Hassan, 2010), and access to the Internet and social networks etc. The demand for the use of these applications is increasing, resulting in the need to ensure the access to them considering minimum quality parameters that can be enforced through a set of Key Performance Indicators KPI (Coffery, 2011). The flexibility and ease of access to the resources offered by applications that run on mobile devices allow obtaining information through the user's own satisfaction with the services offered in the host cities of the mega sporting events.

To provide access to these applications and online services, mega events should have an IT infrastructure that makes it easier for the spectator to use Internet services through dedicated networks, in some cases with signal distribution via Wi-Fi network and via other paid communication services such as the $3 \mathrm{G}$ and $4 \mathrm{G}$ networks.

The locations for mega events also require that cellular phone carriers install transmission antennas in order to maximize, within the area of the event, the quality of data provided by each carrier to its set of customers. As a result of this infrastructure, an extension of applications and useful data for the different areas, such as security, which includes evacuation procedures and crowd control in mega events, can be shared.

Some studies mention the importance of the project and management of large groups, such as urban mobility, presenting a theoretical approach to the life cycle of urban transport models before and after mega sporting events take place (Cheng, 2009a; Cossavelou, 2001). In (Cheng, 2009b), the authors present a set of investments in successful technology for the Beijing Olympics. The study aims to evaluate changes in investment models adopted to contribute to a better development of the technological activities of future Olympic Games. In (Hou, 2006), the study included a model of digital transmission by applying the gatekeeping theory that follows a model of five 
levels to which the authors do not add additional levels, but they believe that new criteria must be applied to an analysis. Variables are composed of 21 questions and the resulting data is processed using an approach to Structural Equation Modeling - SEM.

This paper aims to present the state of the art of a service integration model to mega events. The environment or context of the proposal identifies a division into six subprojects, also called major areas or functional groups. In the telecommunications subproject, in which this paper will be performed, it is described an architecture model of hardware and software to integrate the diversity of services and information, considering a user-centered sharing architecture and also the use of performance indicators to evaluate the service quality provided by the proposed model.

The methodology of validation of the proposal consists in the description and implementation of a case study: the 2014 FIFA World Cup in Brazil, which was divided into two scenarios. The first scenario is already in development and the first version of the second scenario is already in use. Among the contributions, it is considered the facility to identify or to determine KPI indicators for the success of services integration model for mega events.

The paper is organized in the following way: In Section 1, a brief overview of the main related works is presented. In Section 2, a brief view of the mega events and a brief introduction about the project financed by $\mathrm{CNPq}$ is presented. In Section 3, themodel overview is presented. In Section 4, we present a description of the proposed architecture. In Section 5, Operational Architecture. In Section 6, we present a case study, and, finally, in Section 7, the conclusions are presented.

\section{A VIEW OF MEGA EVENTS}

The holding of a Mega Event, whichever their nature, can certainly influence the acceleration of the social infrastructure in progress or encourage a clearer view of the deficiencies of current infrastructure and demand more objective investments.

Growing countries, such as Brazil, has become the target of important Mega Events, like the Confederations Cup, the World Cup and, soon, the Olympics, etc. Thus, there is a clear need not only to measure the impact of the growth of social infrastructure and socioeconomic influences, which, in both cases, we could identify as legacies of the Mega Event, but also to create new ways to acquire, manage, share and represent the information of the environment, which results from the interaction with people.

Specifically in the context of legacies, financing sources and research encouragement, partnerships are initiated with leading academic institutions in the country to study the "Mega Events and their Legacies" phenomenon. The project on which this work is developed is financed by CNPq and seeks to identify the Legacies of Mega Events like the World Cup 2014.

In relation to the overall goal of this project, we must highlight that it is still early for conclusive opinions about holding these Mega Events, their legacy and the population vision in relation to them. This paper focuses on dividing the context into six sub-projects, which, as mentioned earlier, are described as major areas or functional groups (Urban Mobility, Tourism, Airports, Security, Energy and Telecommunications). It aims to analyze not only the legacy for every major area, but also the level of software 
solutions and IT infrastructure in general, to facilitate the integration and communication of the various services provided to viewers inside and outside the sporting environments.

The telecommunication subproject proposes and describes a model of hardware architecture and software to integrate the diversity of services and information, with the user as the basis of an architecture for sharing. It is also considered the use of performance indicators to assess the quality of services provided.

For the development of the proposal, various parameters have been considered to be studied, for example, the high heterogeneity of embedded hardware and communication (Garay, 2013a), protocols devices and the formalization of physical events (Garay, 2013b) that can exist within the context of the proposal.

\section{MODEL OVERVIEW}

The model aims to explore the features and functionalities of mobile devices (mobile phones, tablets, iPads etc.) to obtain information related to major areas or functional groups mentioned in sections I and II.

This information can be resulted from activities related to air transport, safety in public environments (stadiums, museums, parks, subway stations etc.), fire alarms, people location tracking etc., all through an infrastructure of massive sharing of information (Murat, 2010) that can be restricted and subsequently separated as services.

In the context of the proposal, information on each functional group is managed by applications and treated on the level of integration architecture as a set of services, in which each service corresponds to a proper functional context, which has a set of functional capabilities related to this context, for example, safety related to people location tracking inside the stadium, fire control, lighting systems (energy), public transportation, localization and quality of data communication services etc. The capabilities of the set of services must be appropriated to be invoked by external programs ().

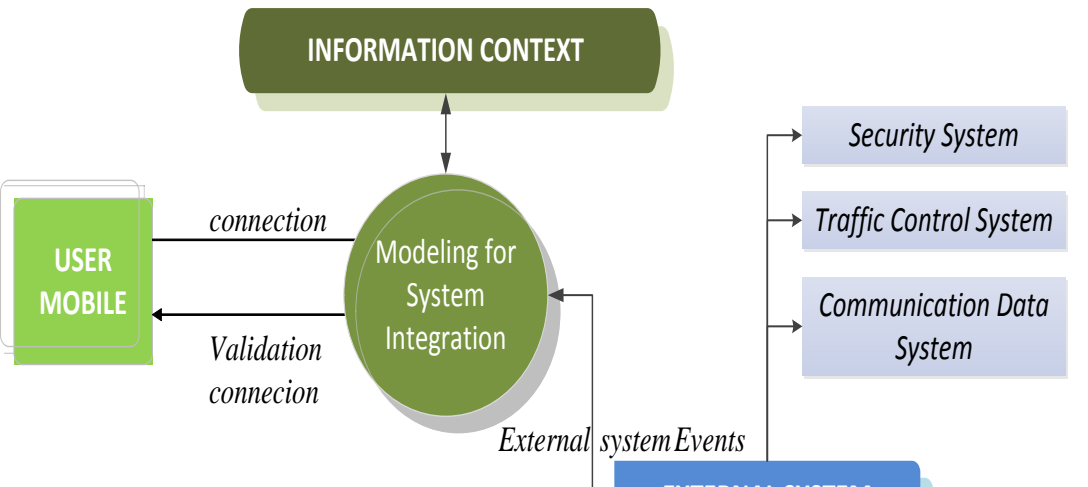

EXTERNAL SYSTEM

Figure 1: DFD of the proposal.

It presents a simplified diagram, however, not less objective, showing the process flow of the proposed architecture, which is described in Section IV. The flow describes four fundamental processes that, according to the extent of modeling, can characterize the complexity of implementation and relevance of each process. 
- $\quad$ Mobile User: It is the term provided to the user set and mobile device. There is, nowadays, a wide range of mobile devices and, as a consequence, the growth of software applications developed is increasing, both for performing simple tasks, like reading, editing, or sharing media (photos, videos), and for controlling automation systems and acquiring context information (real-time and remote). Mobile applications should consider the optimization of some features such as energy (Foll, 2012), when, for example, users take benefit from multiuser applications, such as games, while running some services in the background for acquisition of context information, communication (connection, upload, download ) etc. (Leikas, 2006).

The Mobile User is seen as a process that needs to be abstracted at the level of communication (Wi-Fi, 3G, 4G) and in terms of application processes, being used to make use of a data service or to share information within the same context.

- Modeling System Integration: The creation of functional and structural models and the implementation of knowledge and control data sources (Hsu, 1990) is considered through an approach to manage spatial information (location tracking, time, attribute). This includes metadata templates and a framework (Luo-Yingwei, 2003) to facilitate the interoperability of processes that result from the integration of existing applications between mobile devices and applications that comprise of or result from the organization of events that correspond to each functional area or group.

Service composition techniques (Wan, 2008; Yun, 2013) and events monitoring (Feenstra, 2009) must be considered in the model of system integration, which, as mentioned initially, can act as a framework or as middleware that, in case of seeing the system activities through an interface, can act as a supervisor system.

Context Information: They are the environment information. Currently, environments in which there is a wide availability of mobile devices offer new opportunities to users of these devices to dynamically access resources, information, and services available in the environment (context). Access to these resources, services, data etc. is found by users based on context information characterizing their status and assuming that this information is highly reliable.

In the context in which it accesses or shares a service or feature, the quality of context information plays an important role in improving the offered services (Wei, 2010) and ensuring the correct behavior of applications. In applications for mega events, there are several quality parameters, techniques for information sharing to maintain the safety (Qingsheng, 2007), as well as frameworks and middleware which are needed to integrate applications, services and resources with external systems (Yongkay, 2009), all based on information acquired from the context.

- External System: Context information is needed for the reliable sharing of information and verification of services which are offered during mega events. The resulting set of information is useful to trigger what we call external systems. External systems are applications that correspond to the various departments responsible for the monitoring of mega events. These dependencies can be local, state, federal related to control of mega events in such aspects as security, traffic control, crowd control, fire, energy etc.

The information provided by the user or user group, through a graphical interface, can be classified using semantic techniques along with the creation of ontologies (Key-Sun, 2007) for better classification and representation. 
During the generation of events to trigger external systems there may be a probability that one event is repeated several times under the same conditions that would make access and the external system itself more proactive. The probability can be represented and calculated using the equation (Eq.1):

$$
P_{(A)}=\frac{n_{A}}{N}
$$

Where $P_{(A)}$ is the probability of ${ }_{(A)}$ event to happen, the number of events is ${ }_{A}$, and $N$ is the total number of possible events. To specifically determine the probability of the same event to happen, in order to predict certain events, we must calculate $N$ according to the characteristics and type of event.

\section{ARCHITECTURE DESCRIPTION}

In the proposed model, applications must operate independently due to service architecture with low coupling favoring high application availability and easy maintenance. Each application environment must operate in their own infrastructure, share and integrate information through a distributed process to solve problems related to reliability and availability of services and applications. In Figure 2, the diagram of the integration model is presented, in which the main layers are described objectively here:

- $\quad$ Security Application: This is the first of three main modules implemented at the level of the user interface. In this module, non-functional requirements are considered, such as integrity, availability, and reliability of information for communication with external systems and with user-centered applications.

- Communication Application: This is the second module of the user interface level. Here, the logical model of communication is implemented between the components and the framework, and the physical communication model (infrastructure) between applications, services, and end user.

- $\quad$ Monitoring Application: This is the last of the three modules at the level of user interface and it is in this module that the model is validated through the case study. Non-functional requirements are defined in this module, as well as the modeling of user interface, which includes performance indicators (KPI) of the services that are supported by the integration model.

Requirements: Here, the extension of functional, non-functional, system, and user requirements are defined, modeled and parameterized. The behavior of requirements is defined by the rules of business of each of the applications that will take advantage of the integration model. In the integration layer shown in Figure 2, we present other processes that control activities and information framework and define integration processes to be considered in the implementation of an application, the integration rules and the interfaces. 
- Integration Framework: In this layer, the model of integration is defined by using a design pattern for implementation, component specification, coupling levels, component scalability, dependencies, and, finally, components reusability.

- Service Composed: Commonly, the composition is the reason for something to be decomposed. Something bigger is divided into smaller parts because we identify the potential benefit of being able to do things with these parts that could not be possible if they only existed together. These small parts can be called processes, which we classify into three types: AS (Abstraction Service), CS (Composition Service), and TS (Translate Service).

- Interfaces: They must be developed as a result of the application features and customer needs, allowing interaction between framework, applications, and end user. The user-level interfaces are developed for the Android OS.

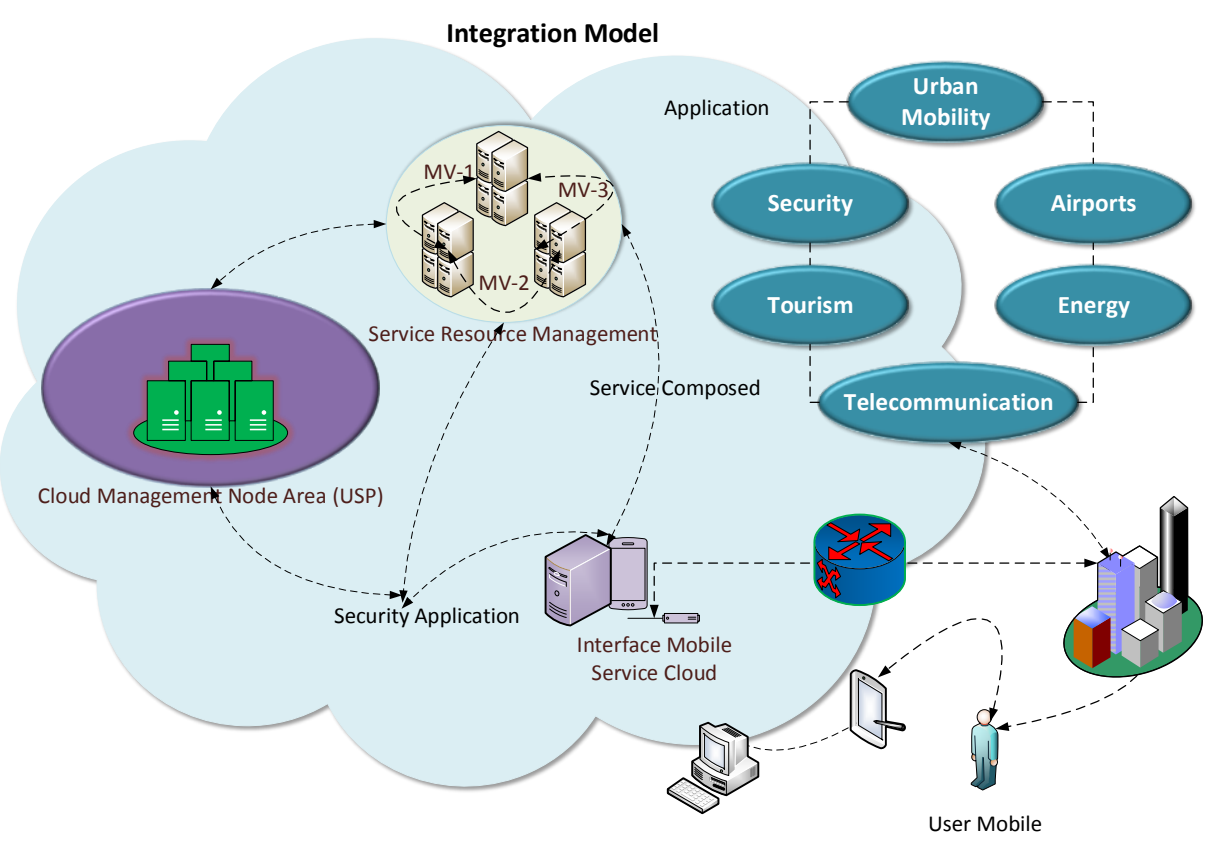

Figure 2: Diagram of the integration model (Framework).

\section{OPERATIONAL ARCHITECTURE}

In this section, we describe the behavior of the layers of the proposed model and the processes involved in the interaction to allow integration, starting from the context information represented by the Functional Groups. The flow behavior of the model is shown in Figure 3 and Figure 4.

In the process of running the operating architecture of the proposed model for the integration of resources, information, and services in mega events, the system performs a mapping from the set of available applications for each functional group, beginning from the communications major area.

From a simplified view, shown in Figure 3, each functional group implements, or can possibly implement, a small or large set of applications and services, which, in turn, can trigger external systems. These applications are integrated into the framework by following a process with predefined business rules. The user will be able to access 
applications via mobile devices using the IMEI (International Mobile Equipment Identity), of their device as the only identifier.

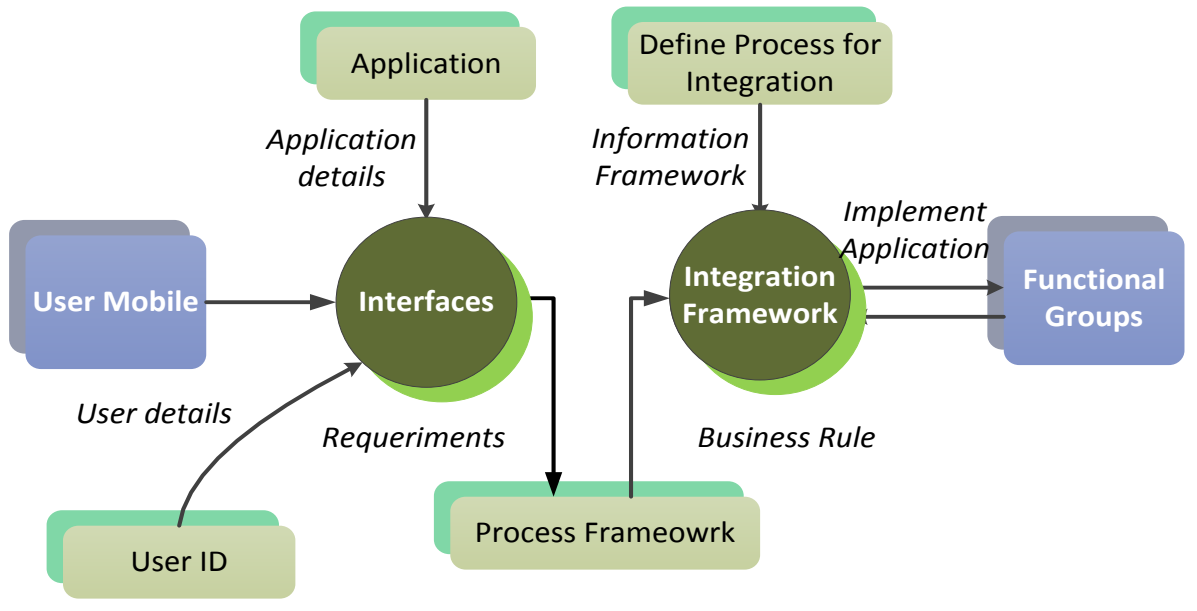

Figure 3: DFD - Integration model of a simplified flow of services.

In the diagram presented in Figure 4, the data and process flow are presented, adding one more level to Figure 3. The functional groups or major areas are also shown in the diagram, all integrated by the Communications area (also known, in the project, as Telecommunications). During service composition, it is necessary to define when, how, and by whom these services will be reused. Business rules determine the use of components, the framework integration with applications, and the validation of the user with the system through an interface available to their mobile device. Finally, all data resulted from applications that belong to each of the major areas are stored in a common data repository, considering that not all information is immediately useful for the various running processes; however, these can be further considered relevant, treated, and represented by a statistical model to determine probabilities and quality indicators of events, using a larger number of details. 


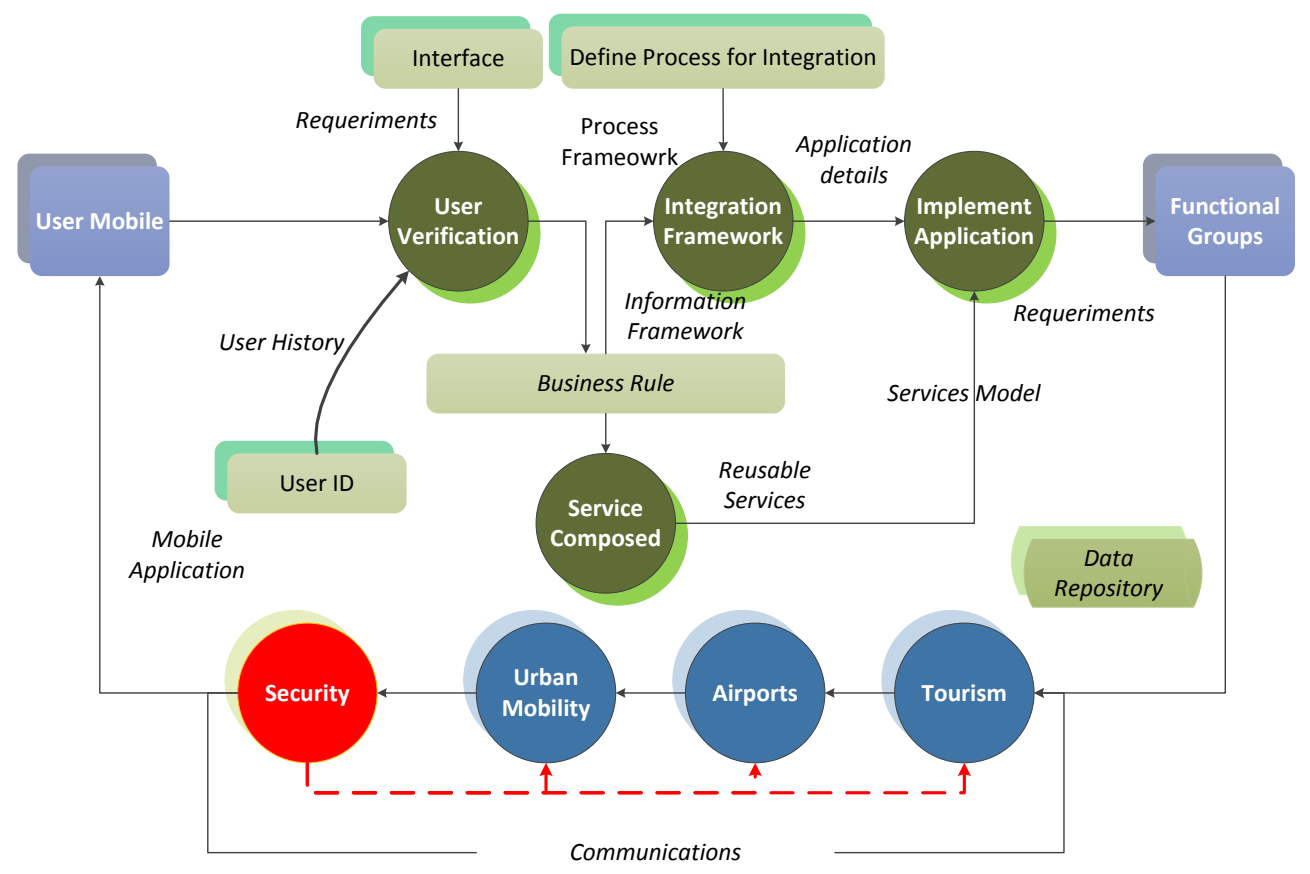

Figure 4: DFD - Detailed flow of service integration model.

\section{CASE STUDY}

The case study for validation of this proposal is based on the description and modeling of a user-centered application. The goal is to supply the necessary tools to provide, to the user, context information that can be used in three processes: The first would be to identify the services provided by the system, the second would be to determine the quality of services purchased, and the third would be to associate information and services to a major area or functional group (Figure 5).

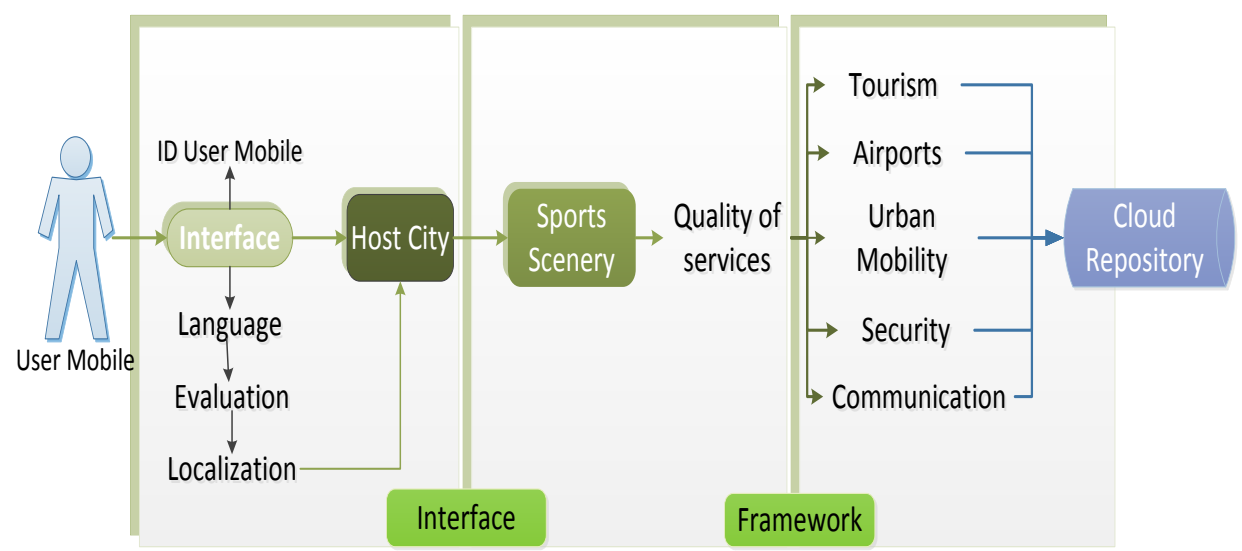

Figure 5: Scenario Diagram for Case Study.

The logic of development (coding) of the proposed model is quite robust given its complexity and the formalism that is considered to measure the quality of each service or set of services, considering indicators (KPI). 
In the case study, two scenarios are described for the implementation. Each scenario represents its own level of complexity; some limitations were identified during the stage of requirements and specification gathering.

\section{- Scenario 1:}

Crowdsourcing; it is considered that many events happen inside of the stadium, and some of these events are related to security, which, in turn, is related to other factors such as lighting, policing, emergency exits, observation of criminal acts, etc. For this specific context, it is necessary to model and implement an environment of mobile application in which the user, when observing any event (relevant or not), is able to share the information of this observation with other users, the ultimate goal would be to improve the quality of security.

The strongest part of the infrastructure model is centered on the user and their ability to share information with other users via their mobile device, primarily in a collaborative environment in which the communication channel would be a wireless network or the Internet itself.

We also consider the exploring of the existing IT infrastructure in the stadiums (Mega Event environments), such as Wi-Fi communication and strong infrastructure implemented by the various phone carriers, to optimize the data connection of their set of customers.

\section{- $\quad$ Scenario 2:}

Indicators, people and Mega Events make up a concrete relationship. People (domestic or foreign tourists) move through the various cities hosting a Mega Event using air or ground transportation and, when they are in the city, they use services such as public (subway, bus) or private (rental vehicles or taxi) transportation and take advantage of the major tourist locations. In this cycle, they observe important elements, such as traffic conditions, quality of transport, facility for getting around, lodging, street lighting, policing, perception of safety in the city, in the stadium or in any other environment where they are attending the event, and etc. This set of information is relevant to create indicators, not only about the quality of a service, but also to determine the impact of a direct or indirect legacy of the Mega Event.

A robust relationship of indicators, location and host cities is modeled for this scenario. This part of the proposal begins by checking in which of the host cities the user is using their mobile device's GPS. After this observation, we present a set of interfaces that guide the user through a collaborative process, with questions about the quality of infrastructure and services. When the user's location is identified, the application presents airports, bus terminals, subway stations, stadiums and events (games) that will be performed exclusively in that location. An important aspect is that the user does not have access to indicators or services of the mobile application if they are not in one of the host cities of the Mega Event, thus creating a relationship of reliability and integrity of the collected information.

The Mega Event needs to be measured always considering their impact on the services offered to the people (tourists and locals). This impact can be extended even to social infrastructure (legacy), a Mega Event's exclusive infrastructure and infrastructure 
and services that were accelerated by the occurrence of the Mega Event. To complete this process, it is necessary to use performance indicators.

\section{- $\quad$ Performance Indicators}

The KPI is composed of the following indicators: Availability $\left(D_{i}\right)$, Opportunity $\left(O p_{i}\right)$, Information $\left(\mathrm{In}_{i}\right)$, and Reliability $\left(\mathrm{Co}_{i}\right)$. The weight of each indicator can change according to the services conditions that are offered by each functional group, for example, the weight assigned to the indicator reliability can switch to the services provided within the functional group of urban mobility, where, at certain times, the flow of vehicles can change as a result of having a greater number of people, $D_{i}$ may be lower as well as $O p_{i}$ to access the same means of transport.

$$
K P I_{U M}=\sum_{i=1}^{n} w_{i} \cdot I_{i}
$$

Where $\sum_{i=1}^{a} w_{i} \cdot I_{i}$ is the sum of the products of the weight of each indicator for the value of the corresponding indicator is, $W_{i}$ is the weight or weighted value of each indicator and $\mathrm{Ii}$ is the value of each indicator.

Under typical conditions (static), $W_{i}$ values are considered constant according to the historical development of the service, and, in scenarios of special conditions, it can be dynamically calculated using the following expression, which represents a situation of proportional distribution (Eq. 3):

$$
w_{i}=\frac{100 \cdot I_{i}}{\sum_{i=1}^{n} I_{i}}
$$

Where to all the weight of the weighting factor there is a correspondent, there is the value of the indicators that is established by the conditions of the special scenario, and the total value of indicators.

When considering the four indicators mentioned initially, the expression (Eq. 2) can be represented in (Eq. 4):

$$
K P I_{U M}=w_{1} \cdot D_{1}+w_{2} \cdot O p_{2}+w_{3} \cdot I n_{3}+w_{4} \cdot \mathrm{Co}_{4}
$$

\section{- Cluster environment for the Cloud}

The hardware that will be used corresponds to anIBM architecture, strictly a Blade Center with two HS22V blades (Xeon 6C X5650 - $2.66 \mathrm{GHz}$ with $144 \mathrm{~GB}$ of RAM) and four HS22 blades with support for 96GB RAM for virtualization. An IBM Director Server (Xeon 4C E5620 - $2.40 \mathrm{GHz}$ ) and a DS3500 Storage with 19 SAS disk of $600 \mathrm{~GB}$. 


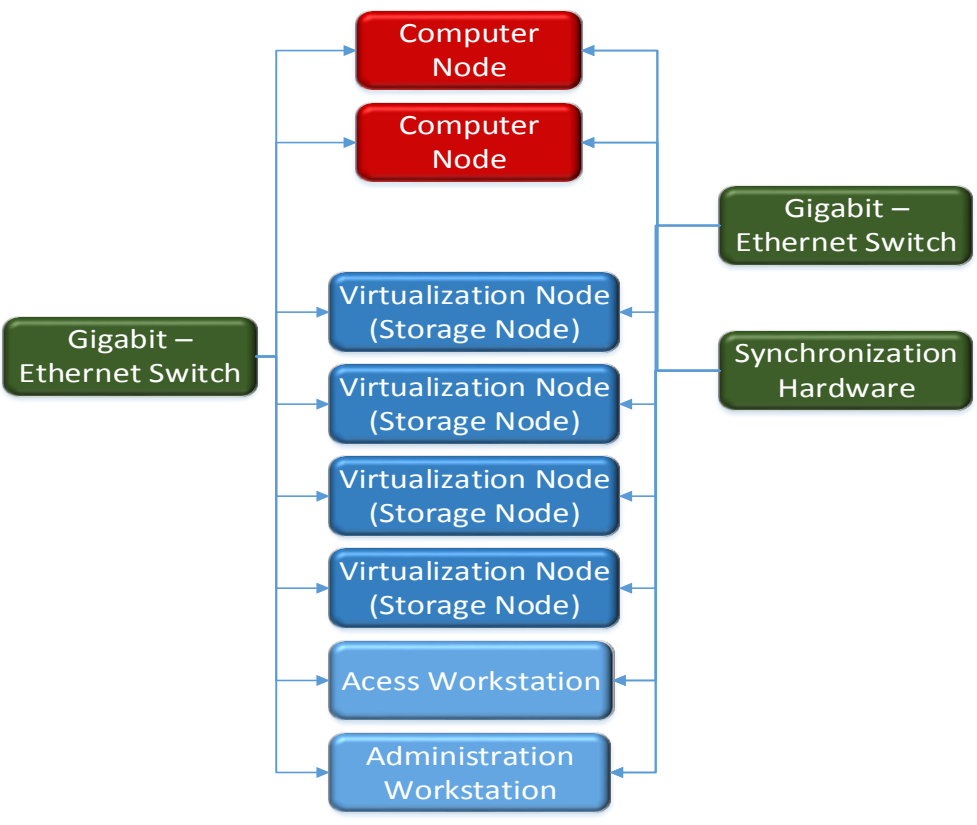

Figure 6: Cluster architecture - IBM Cloud.

The IBM BladCenter is a very robust architecture and it is available in CITI (Interdisciplinary Center for Interactive Technologies), from the University of São Paulo (USP), which is why CITI has been the ideal choice to meet a high flow of requests and data storage. It also allows the rationalization of resources when less hardware is required, reducing the short running costs and energy use, which is an ideal situation since it is considered that it should meet, after system deployment, a high flow of requests, but for short time periods.

The system virtualization must ensure a perfect circle of lower power consumption and performance, helping to ensure that the high data and requests flow (many events that result from the applications developed for each functional group) is not a factor that impacts on the operating system reliability. Virtualization has opened the way to new organizational models, such as cloud computing, allowing services to be easily deployed considering the condition that a continuous monitoring system has been implemented in the background.

\section{- Environment for performing experiments}

The environment for performing system tests (Figure 7) is modeled to ensure correct coding and fewer errors in the data structure, and also to meet the requirements of software quality. The test environment will allow us to mainly eliminate loading errors and to reduce the approval of new applications to be integrated in proposed model during the integration of services considering the extension of each area or functional group within the context of mega events, especially considering the high workload and the behavior of the model under normal conditions.

The performance test should start by the web monitoring, hardware monitoring, availability, and response time to service requests and catching errors. 


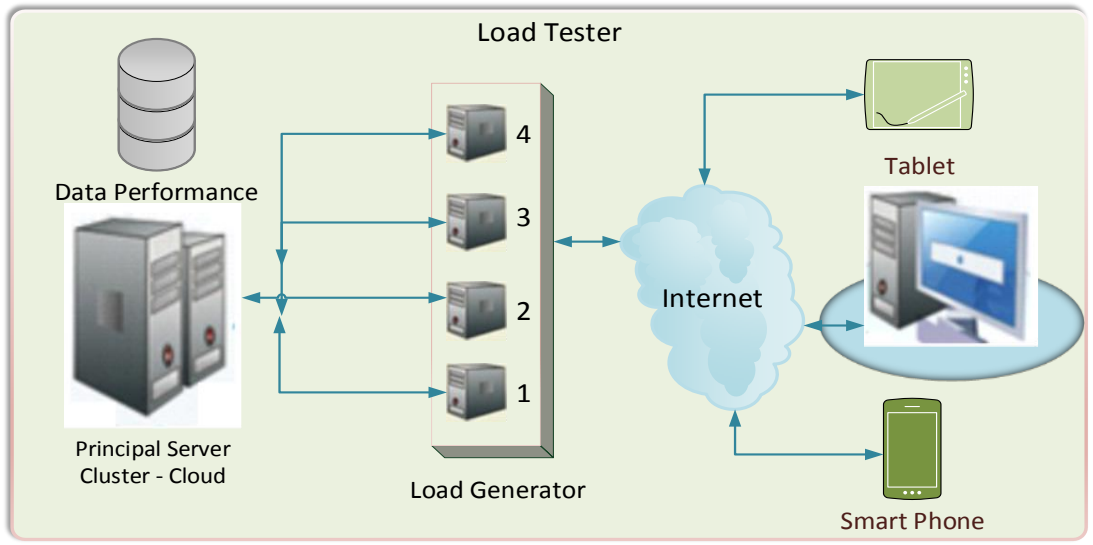

Figure 7: Environment for tests.

Another parameter that must be analyzed over the model is the scalability. In this context, scalability means the responsiveness of the system in relation to resource demand that is required from it. It is considered that, in mega sporting events, the data flow generated by applications that will be integrated into the framework for later storage in the data structure must be pretty high.

\section{CONCLUSIONS}

The number of researches and projects performed to develop or propose to manage the hardware infrastructure, applications and information derived from the mega events context is limited, usually to the environment in which the event happens. Details on the procedures performed by a framework or middleware in application integration are largely unknown since in most cases the solution is specific and proprietary.

This paper conducted a detailed survey of the main contributions in the mega events area and tried to base the model as well as the use of performance indicators.

Although the approach seems to be very comprehensive and, at some points, subjective, it is intended that the descriptions of the layers and processes of the model are the point of reference for the development of applications and services for mega events.

In the proposal, we intend to present a model that is consistent with the needs of the project financed by the National Council of Technological and Scientific Development - CNPq, with the goal of creating solutions to manage architectures, applications and information flow in mega events.

The processes and activities described in each scenario should be considered relevant to hold any mega event and, on this matter, there is a clear tendency to create highly complex applications, favoring the representation of information and control interaction between physical events and applications (Garay, 2010c). New concepts and methods need to be explored aiming to create a single integrated system of information and control events such as security, telecommunications, traffic, urban transport in real time, etc. 
The first version of the implementation of the second scenario is available on Google Play and its implementation methodology has been accepted for publication in (Garay, 2014d) and the version, in its view of software product in (Garay, 2014e). The application presents important platform features for application of performance indicators that allow the measure of social impact on services social infrastructure in each of the mega event host cities.

We must mention the difficulty accessing the information that would enable a more complete pilot solution, which includes the implementation of the first scenario. The difficulty originates from the resistance of the stadium managers, major focus of the mega event, in providing access to technical information. They would have even favored the planning of the nonexistent communication infrastructure of most stadiums hosting the World Cup in 2014.

\section{ACKNOWLEDGMENT}

The authors would like to thanks CNPq (Conselho Nacional de Desenvolvimento Científico e Tecnológico) that offered support for this work by project Encomendas ME/CNPq - Legados e oportunidades Gerados pela Copa do Mundo 2014 under process number 400054/2013-2 and to the Interdisciplinary Center in Interactive Technologies of University of Sao Paulo - (CITIUSP).

\section{REFERENCES}

Cheng Xiaoduo, "The Interaction Mechanism between Olympics' Venues and Urban System," Management and Service Science, 2009. MASS '09. International Conference on , vol., no., pp.1,4, 20-22 Sept. 2009.

Cheng Xiaoduo, "The Urban System Impact on Post-Games Development of the Olympics' Venues in China," Computer Science and Information Technology - Spring Conference, 2009. IACSITSC '09. International Association of , vol., no., pp.397,402, 17-20 April 2009.

Coffey, V.; Willar, D.; Trigunarsyah, B., "Quality management system and construction performance," Quality and Reliability (ICQR), 2011 IEEE International Conference on , vol., no., pp.403,407, 14-17 Sept. 2011. doi: 10.1109/ICQR.2011.6031750.

Cossiavelou, V.; Bantimaroudis, P.; Kavakli, E.; Illia, L., "Wireless communications in Olympic Games: R\&I into the frame of the updated gatekeeping model," Wireless Telecommunications Symposium (WTS), 2011 , vol., no., pp.1,5, 13-15 April 2011.

Feenstra, R.; Janssen, M., "Service Composition in Public Networks: Results from a Quasi-Experiment," System Sciences, 2009. HICSS '09. 42nd Hawaii International Conference on , vol., no., pp.1,10, 5-8 Jan. 2009. doi: 10.1109/HICSS.2009.385.

Foll, S.; Herrmann, K.; Rothermel, K., "Energy-Efficient Update Protocols for Mobile User Context," Advanced Information Networking and Applications (AINA), 2012 IEEE 26th International Conference on, vol., no., pp.120,127, 26-29 March 2012. doi: 10.1109/AINA.2012.129.

Garay, J.R.B.; De Oliveira A. M., Kofuji, S.T., "Proposed Middleware for Sensor Networks in Cyber-Physical System Environments", in Proc. SENSORCOMM 2013 : 
The Seventh International Conference on Sensor Technologies and Applications. pp. 178-185, 2013.

Garay, J. R.B; De Oliveira A. M. Kofuji, S.T., "Cyber-Physical Systems (CPS): Description of a Solution to Heterogeneous Architectures Management", in Proc. LATINCOM 2013: 5th IEEE Latin-American Conference on Communications 2013.

Garay, J.R.B.; Kofuji, S.T., "Architecture for sensor networks in cyber-physical system," Communications (LATINCOM), 2010 IEEE Latin-American Conference on , vol., no., pp.1,6, 15-17 Sept. 2010. doi: 10.1109/LATINCOM.2010.5641126

Garay, J.R.B, Calixto G. M, De Oliveira A. M., Zuffo M. K." Performance Indicators: A Mobile Solution to Identify Legacies in Mega Events", The 4th IEEE International Conference on Consumer Electronics - Berlin (IEEE-ICCE-Berlin). 2014.

Garay, J.R.B, Calixto G. M, De Oliveira A. M., Zuffo M. K." Mobile Applications and Legacies of Mega Events", in Proc. INTERCON 2014: International Congress in Electronic, Electrical and Engineering. vol. 21 pp. 315-319. Perú. 2014.

Hasan, S.I.; , "Multiplayer gaming for low-end mobile phones: Gaming between basic mobile phones, handheld devices and computer platforms," Games Innovations Conference (ICE-GIC), 2010 International IEEE Consumer Electronics Society's , vol., no., pp.1-5, 21-23 Dec. 2010.

Hou Yu-peng; Ju Xiao-feng, "The Study on the Influence Channel and the Degree of Impact of Olympic Economy of Beijing," Management Science and Engineering, 2006. ICMSE '06. 2006 International Conference on, vol., no., pp.1145,1150, 5-7 Oct. 2006. doi: 10.1109/ICMSE.2006.314204

Hsu, C.; Bouziane, M.; Cheung, W.; Nogues, J.; Rattner, L.; Yee, L., "A metadata system for information modeling and integration," Systems Integration, 1990. Systems Integration '90., Proceedings of the First International Conference on, vol., no., $\begin{array}{llll}\text { pp.616,624, } & \text { 23-26 } & \text { Apr } & 1990\end{array}$ doi: $10.1109 /$ ICSI.1990.138727

Key-Sun Choi, "IT Ontology and Semantic Technology," Natural Language Processing and Knowledge Engineering, 2007. NLP-KE 2007. International Conference on , vol., no., pp.14,15, Aug. 30 2007-Sept. 1 2007. doi: 10.1109/NLPKE.2007.4368004

Leikas, J.; Stromberg, H.; Ikonen, V.; Suomela, R.; Heinila, J., "Multi-user mobile applications and a public display: novel ways for social interaction," Pervasive Computing and Communications, 2006. PerCom 2006. Fourth Annual IEEE International Conference on, vol., no., pp.5 pp.,70, 13-17 March 2006. doi: 10.1109/PERCOM.2006.38

LuoYingwei; Wang Xiaolin; Xuzhuoqun, "Spatial semantic network and agent-based framework for spatial information interoperation," Information Reuse and Integration, 2003. IRI 2003. IEEE International Conference on, vol., no., pp.40,47, 27-29 Oct. 2003. doi: 10.1109/IRI.2003.1251393

Murat Demirbas, Murat Ali Bayir, CuneytGurcanAkcora, YavuzSelimYilmaz, and HakanFerhatosmanoglu. 2010. Crowd-sourced sensing and collaboration using twitter. InProceedings of the 2010 IEEE International Symposium on A World of Wireless, Mobile and Multimedia Networks (WOWMOM '10). IEEE Computer Society, Washington, DC, USA, 1-9. 
N. Eagle and A. Pentland, "Social serendipity: Mobilizing social software," IEEE Pervasive Computing, vol. 04-2, pp. 28-34, 2005.

Qingsheng Zhang; Yong Qi; Jizhong Zhao; Di Hou; Tianhai Zhao; Liang Liu, "A Study on Context-aware Privacy Protection for Personal Information," Computer Communications and Networks, 2007. ICCCN 2007. Proceedings of 16th International Conference on, vol., no., pp.1351,1358, 13-16 Aug. 2007. doi: 10.1109/ICCCN.2007.4318009

Rose, R.; Meier, C.; Zorn, S.; Goetz, A.; Weigel, R.; , "A GSM-network for mobile phone localization in disaster scenarios," Microwave Conference (GeMIC), 2011 German , vol., no., pp.1-4, 14-16 March 2011.

Wan Fang; Shen Jianjing; Han Ding; Zhang Hui, "On composition of service component based on service-oriented architecture," Control Conference, 2008. CCC 2008. 27th Chinese, vol., no., pp.573,576, 16-18 July 2008 . doi: 10.1109/CHICC.2008.4605721

Wei Zhang; Chang, C.K.; Taiming Feng; Hsin-yi Jiang, "QoS-Based Dynamic Web Service Composition with Ant Colony Optimization," Computer Software and Applications Conference (COMPSAC), 2010 IEEE 34th Annual, vol., no., pp.493,502, 19-23

doi: 10.1109/COMPSAC.2010.76

July

2010

Yongkai Zhan; Shuangquan Wang; Zhuang Zhao; Canfeng Chen; Jian Ma, "A mobile device oriented framework for context information management," Information, Computing and Telecommunication, 2009. YC-ICT '09. IEEE Youth Conference on, vol., no., pp.150,153, 20-21 Sept. 2009. doi: 10.1109/YCICT.2009.5382404

Yun Ma; Xuanzhe Liu; Yihan Wu; Grace, P., "Model-Based Management of Service Composition," Service Oriented System Engineering (SOSE), 2013 IEEE 7th International Symposium on, vol., no., pp.103,112, 25-28 March 2013. doi: 10.1109/SOSE.2013.21 